\title{
Prenatal diagnosis in CDG1 families: beware of heterogeneity
}

\author{
G ert M atthijs ${ }^{1}$, Els Schollen ${ }^{1}$, J ean-Jacques C assiman ${ }^{1}$, Valérie Cormier-D aire ${ }^{2}$, \\ Jaak Jaeken ${ }^{3}$ and E mile van Schaftingen ${ }^{4}$ \\ ${ }^{1}$ Center for $\mathrm{H}$ uman $\mathrm{G}$ enetics, U niversity of L euven, B-3000 L euven, B elgium \\ ${ }^{2}$ D epartment of G enetics, IN SE RM U.393, H ôpital des E nfants M alades, 75743 Paris, France \\ ${ }^{3}$ D epartment of Paediatrics, U niversity of L euven, B-3000 L euven, B elgium \\ ${ }^{4} \mathrm{~L}$ aboratory of Physiological Chemistry, ICP and U niversity of $L$ ouvain, $B-1200$ Brussels, B elgium
}

\begin{abstract}
Carbohydrate-deficient glycoprotein syndrome type I (CDG1) is an autosomal recessive, metabolic disorder with severe psychomotor retardation and a high mortality rate in early childhood. Most patients have a deficiency of phosphomannomutase, due to mutations in PMM2, a gene located on chromosome 16p13. Over a period of 18 months we offered prenatal diagnosis to eight families. In six cases and prior to the identification of the gene, the diagnosis was based on linkage analysis and phosphomannomutase measurements. Subsequently direct mutation analysis has been used in two families. It is shown here that phosphomannomutase activities are strongly reduced in cultured amniocytes and trophoblasts of affected foetuses. We refrained from offering prenatal testing in two other families, because either the disease did not link to chromosome 16 and/or normal phosphomannomutase activities were measured in fibroblasts from the proband. This confirms earlier suggestions of heterogeneity for CDG1.
\end{abstract}

Keywords: Jaeken syndrome; phosphomannomutase; recessive disease; chromosome 16; mutation analysis; linkage analysis; amniocytes; trophoblasts

\section{Introduction}

Carbohydrate-deficient glycoprotein syndrome type I (CD G 1) or Jaeken disease (O M IM 212065) is a genetic multi-system syndrome. At birth, the patients have a peculiar abnormal distribution of subcutaneous fat and nipple retraction. There is encephalopathy with axial hypotonia, abnormal eye movements, internal stra-

Correspondence: $\mathrm{G}$ ert $\mathrm{M}$ atthijs, $\mathrm{C}$ enter for $\mathrm{H}$ uman $\mathrm{G}$ enetics, C ampus $G$ asthuisberg $O \& N$ 6, H erestraat 49, B -3000 L euven, B elgium

R eceived 28 J uly 1997; revised 140 ctober 1997; accepted 22 O ctober 1997 bismus, pronounced psychomotor retardation without regression, as well as peripheral neuropathy, cerebellar hypoplasia and retinitis pigmentosa. Severe infections, liver insufficiency or cardiomyopathy lead to $20 \%$ lethality in the first years of life. ${ }^{1,2}$

The disease is associated with defective glycosylation of glycoconjugates. ${ }^{3-6}$ The diagnosis of CDG 1 has classically been based on the abnormal pattern which is observed after iso-electric focusing (IEF) of serum transferrins. ${ }^{3,4} \mathrm{U}$ nfortunately, the transferrin assay does not reveal an aberrant pattern in amniotic fluid or in foetal blood. ${ }^{7,8}$ Since the CD G 1 locus has been mapped to chromosome $16 \mathrm{p},{ }^{9}$ prenatal diagnosis has become 
possible by linkage analysis. H owever, a major concern has arisen with the identification of genetic heterogeneity in CDG 1. It was initially shown that the disease did not link to chromosome $16 \mathrm{p} 13$ in a family with two affected siblings, ${ }^{10}$ and we describe another case in the present report. O bviously, a prenatal test with linked genetic markers on chromosome $16 p$ is of no value in such cases.

In 1995, Van Schaftingen and Jaeken identified a deficiency of phosphomannomutase activity in patients with CDG $1 .^{11}$ We have recently identified PMM 2, a phosphomannomutase gene on chromosome $16 \mathrm{p} 13 .^{12}$ Mutations in this gene in CDG1 patients provide substantial evidence that it is the gene for CDG 1 . The phosphomannomutase deficiency has now been found in more than 50 CDG 1 patients from various geographical origin, ${ }^{13}$ and mutations have been identified in a majority of these patients. ${ }^{14}$ Thus a direct test is now available for prenatal diagnosis.

$\mathrm{H}$ ere we report on prenatal diagnosis in eight CDG 1 families, thereby showing that phosphomannomutase activities are reduced in amniocytes and trophoblasts of affected foetuses. We would like to spread a cautionary note on the use of linked genetic markers or phosphomannomutase measurements in the prenatal diagnosis of CDG 1 in cases in which the diagnosis has not been confirmed by phosphomannomutase measurements or mutation analysis in the proband or in the parents.

\section{Materials and Methods}

\section{Linkage Analysis}

Polymorphic CA -repeats in the interval between D16S405 and D16S406 were amplified from genomic DNA from lymphocytes, fibroblasts, or cultured amniocytes. ${ }^{9,10}$ M arkers D $16 S 3020$ and D $16 S 3087$ have only recently been mapped to the interval between D 165513 and D 16S404. ${ }^{15}$

\section{Phosphomannomutase Activities}

Phosphomannomutase was assayed spectrophotometrically. ${ }^{13}$ In this report, the enzymatic activities are expressed as a percentage of the mean values in controls. Control activities were (means $\pm S D) 2.46 \pm 0.48 \mathrm{mU} / \mathrm{mg}$ protein $(\mathrm{n}=12$ ) in leukocytes; $3.77 \pm 0.86(n=8)$ in fibroblasts; and $6.7 \pm 1.0$ $(n=3)$ in amniocytes.

\section{Mutation Analysis of PMM2}

Single-strand conformation polymorphism (SSCP) analysis was used to identify mutations in the PMM 2 gene, and the underlying base substitutions were identified by sequencing, as described. ${ }^{12} \mathrm{~A}$ complete set of primers for the analysis of PMM 2 will be described elsewhere. ${ }^{14}$

\section{Results}

The proband in family 15 (Figure 1a) has a phosphomannomutase deficiency with a value below $0.1 \mathrm{mU} / \mathrm{mg}$ protein in fibroblasts (HS in Table 1 in Van Schaftingen and Jaeken). ${ }^{11}$ DNA analysis with linked genetic markers on cultured amniocytes revealed that the foetus was not affected but was a heterozygous carrier. Phosphomannomutase measurements on these amniocytes showed intermediate values, compatible with a carrier status. A healthy baby was born, as expected.

In family 37 , a crossover within the $C D G 1$ region between D 16S414 and D 16S407 was observed on the maternal chromosome 16 in the patient (Figure 1b). Based on published data, the test with the genetic markers was inconclusive. However, the phosphomannomutase measurements showed that the foetus was affected (less than $2 \%$ of the normal values) and these data were considered conclusive. This is consistent with more recent mapping data: the PM M 2 gene is located between D16S406 and D 16S3078 ${ }^{16}$ (this location is more telomeric than we had previously inferred from association data, ${ }^{10}$ and is in accordance with the data from the Scandinavian group.,17 The pregnancy was terminated. Foetal tissue was obtained and assayed for phosphomannomutase: the activity was $<0.05 \mathrm{mU} / \mathrm{mg}$ protein in muscle and liver.

In a third family with a phosphomannomutase deficiency in the proband and evidence for linkage to chromosome $16 p 13$ (family 1 in $M$ atthijs et al ${ }^{10}$ the proband and foetus have inherited a different paternal chromosome (not shown). However, direct phosphomannomutase measurements on cultured amniocytes revealed low values (20\% of mean control values), which could either be interpreted as a (partial) deficiency or a heterozygotic value. A s the genetic data are not compatible with a disease state, the latter was withheld. The low values were attributed to the poor growth of amniocytes. The diagnosis in this case thus mostly relied on the genetic data. A healthy baby was born.

In a fourth family, we refrained from offering a prenatal test because fibroblasts from the proband showed normal phosphomannomutase values. The marker data also suggested that linkage to chromosome 16 p13 was unlikely (family 18 , Figure $1 c$ ). The absence of linkage to the CDG 1 locus was confirmed after the birth of a second affected child in this family.

A prenatal diagnosis was offered to three other families with a known phosphomannomutase deficiency (families 2, 9 and 32, data not shown), and in all cases 


\section{A family 15}

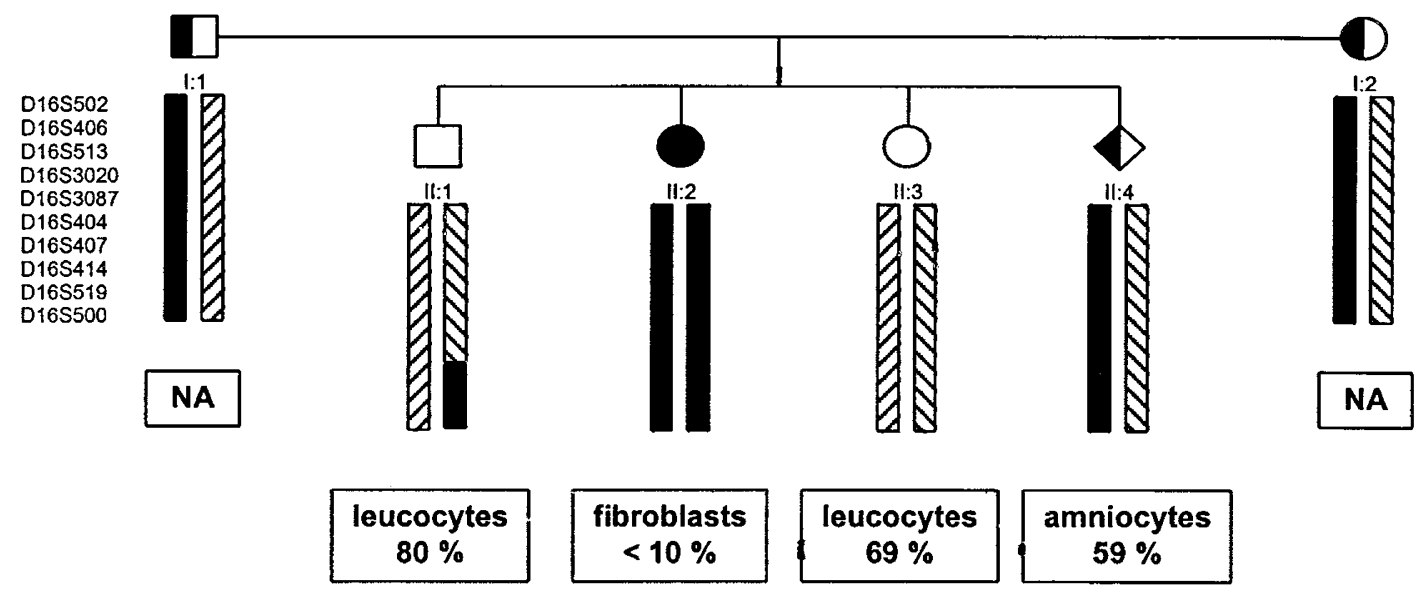

\section{B family 37}

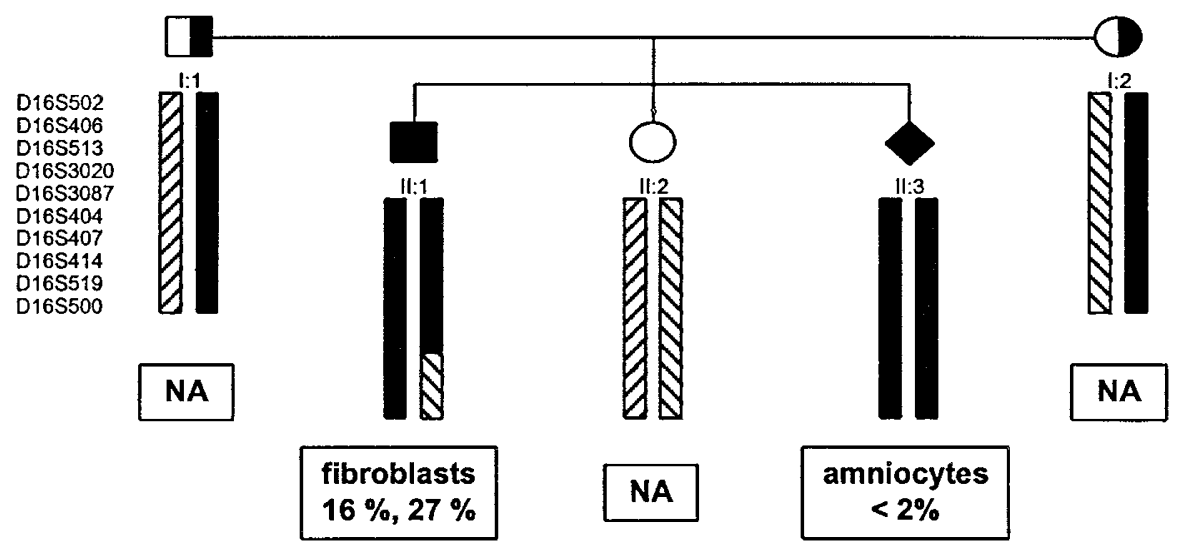

\section{C family 18}

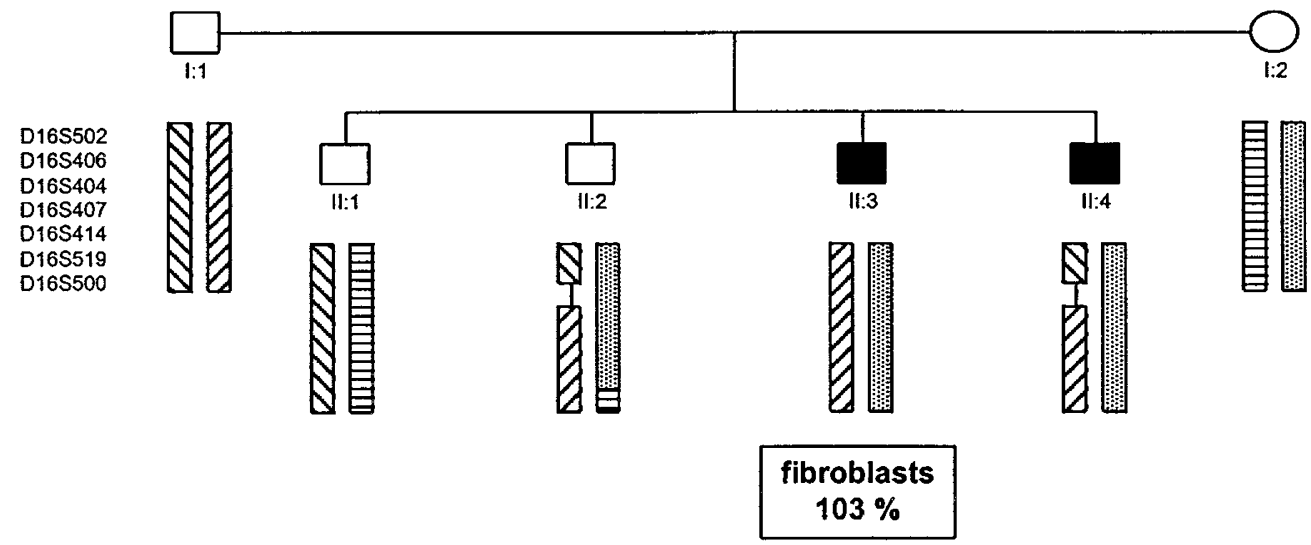

Figure 1 Schematic presentation of three CDG 1 families with marker data and phosphomannomutase (PMM) results.

The polymorphic markers are ordered from telomeric to centromeric, top to bottom. The dark chromosome is associated with the disease. In family 18, the disease is not linked to chromosome 16. E nzymatic activities are expressed as a \% mean values in controls. $\mathrm{NA}=$ no phosphomannomutase data 
the biochemical and genetic data were consistent. $\mathrm{H}$ ealthy babies were born (one is predicted to be a carrier). By now, P M M 2 mutations have been identified in these families. ${ }^{14}$

More recently, prenatal diagnosis was based on the direct mutation analysis of the PM M 2 gene in families in which the mutations had been identified. In family 30 , the proband has the A $108 \mathrm{~V}$ and the common $\mathrm{R} 141 \mathrm{H}$ mutations, as shown by SSCP analysis and confirmed by sequencing. ${ }^{12}$ SSCP analysis of DNA isolated from cultured trophoblasts revealed that the foetus had inherited the paternal $\mathrm{R} 141 \mathrm{H}$ mutation but not the maternal A108V mutation (Figure 2). A phosphomannomutase value of $1.2 \mathrm{mU} / \mathrm{mg}$ protein was compatible with the carrier status. In family 45, no material was available from the deceased proband. However, phosphomannomutase measurements on leukocytes of the parents revealed that they were carriers, which was confirmed by mutation analysis (data not shown): the father carries the F119L mutation and the mother the $\mathrm{R} 141 \mathrm{H}$ mutation, and by deduction, the proband had the $\mathrm{F} 119 \mathrm{~L} / \mathrm{R} 141 \mathrm{H}$ genotype. Both mutations were found in the foetus. Phosphomannomutase values of $0.35 \mathrm{mU} / \mathrm{mg}$ protein in cultured amniocytes confirmed the affected status. In family 44 , in which the proband did not show a phosphomannomutase deficiency, prenatal testing was not possible.

\section{Discussion}

A $n$ unfortunate result in prenatal diagnosis was obtained when, a few years ago, Clayton and collaborators used the transferrin assay on foetal blood. ${ }^{7}$ A pparently, the IEF pattern is normal in foetal blood and soon after birth, an observation that remains unexplained to date.

In 1994, Martinsson and collaborators mapped the CDG 1 candidate region to chromosome 16p13, to a region of $13 \mathrm{cM}$ between markers D16S406 and D 16S405. ${ }^{9}$ Thus an indirect genetic test became available, based on the analysis of linked markers. H owever,



Figure 2 Direct mutation analysis of the PMM 2 gene

PCR amplification of exon 4 (left panel) and exon 5 (right panel) of the PM M 2 gene, that harbour the A $108 \mathrm{~V}$ and R $141 \mathrm{H}$ mutations respectively (see ${ }^{12}$ ), on genomic D NA from the patient, parents and foetus, was followed by SSCP analysis. The bands associated with the mutation are indicated with an arrowhead. The A $108 \mathrm{~V}$ mutation is of maternal origin in this family. The $\mathrm{R} 141 \mathrm{H}$ mutation is inherited from the father. The foetus inherited the paternal disease allele, and is a carrier of the R $141 \mathrm{H}$ mutation. 
the observation of genetic heterogeneity for $C D G 1^{10}$ also jeopardised this assay.

A major breakthrough for CD 1 was the identification of a phosphomannomutase deficiency in patients. ${ }^{11}$ In this report, we show that phosphomannomutase is deficient in (cultured) trophoblasts and amniocytes from affected foetuses. In eight families, linkage or mutation analysis was combined with phosphomannomutase measurements of foetal cells. The results suggest that low to intermediate values should be interpreted with caution. Low values were obtained in poorly growing amniocytes in family 1 , even though linkage analysis predicted a normal genotype. N ote also that there are important variations in the phosphomannomutase values obtained in different cell types from patients with the same genotype. For instance, in fibroblasts from the proband in family 37 (see Figure 1), activities of up to $27 \%$ of normal values were obtained in two independent assays. H owever, in the amniocytes from the affected foetus in the same family, phosphomannomutase values were virtually zero, whereas both patients share the same genotype. It might well be that the phenotype is partly rescued in cells after prolonged culture of the fibroblasts; however, in no instance have we measured in fibroblasts from patients values above $30 \%$ of the normal values.

The problem of interpretation of biochemical data or the risk of recombination between the markers and the mutation is solved by direct mutation analysis, provided that the paternal and maternal mutation have been identified. We presented the results for two such cases. Taken together, we predicted two affected foetuses and three carriers of either a paternal or maternal allele, while the remaining three foetuses did not inherit a disease chromosome. Given the sample size, this is consistent with the autosomal recessive inheritance of the disease. It is now also clear that some patients with the characteristically abnormal IEF pattern of serum transferrins, do not have a phosphomannomutase deficiency ( ${ }^{13}$ and the present report). Most likely, however, in all families with a phosphomannomutase deficiency in the proband, there is linkage to the CD G 1 locus on chromosome $16 \mathrm{p} 13$.

In conclusion, the present results show that prenatal diagnosis for CDG 1 can now reliably be made. Our recommendations for the prenatal diagnosis of CDG 1 are as follows. O ne should first determine the phosphomannomutase activity in fibroblasts, leukocytes or lymphocytes of the proband, or if not possible, in leukocytes of the parents, and look for mutations in the
PM M 2 gene. If no phosphomannomutase deficiency is found, no prenatal diagnosis should be offered at this stage. If a phosphomannomutase deficiency is found and the mutations are identified, the prenatal diagnosis should primarily be based on the detection of mutations. If the mutations are not found, phosphomannomutase measurements should be combined with linkage analysis.

\section{Acknowledgements}

We thank $\operatorname{Dr} M$. Fohlen (Hôpital Saint Vincent de Paul, Paris) and Dr E. Gautier (A merican Hospital, Paris), Dr A. G ouget (H ôpital Saint-J acques, B esançon) and D r T. Simeray (Centre H ospitalier G énéral, L ons-L e-Saunier), D r E . L egius (Centrum voor Menselijke Erfelijkheid, Leuven), D r W. R head (U niversity of I owa, I owa City, IA), Dr N. Seta (H ôpital Bichat, Paris), D r M . van Der K naap (A cademisch Z iekenhuis, V rije U niversiteit, A msterdam), and $\mathrm{Dr} R$. Wevers and D r C. van der B urght (A cademisch Ziekenhuis, $\mathrm{Nijmegen)}$ for referring families and samples for prenatal diagnosis.

We are indebted to K. Minner, G. Berghenouse and G. $D$ eferme for mutation analysis, phosphomannomutase measurements and cell culture.

This work was supported by the B elgian Federal Service for Scientific, Technical and Cultural A ffairs, by the 'A ction de Recherche Concertées' and by the National Fund for Scientific R esearch, B elgium.

\section{References}

1 Jaeken J, Carchon $\mathrm{H}$ : The carbohydrate-deficient glycoprotein syndromes: an overview. J Inher M etab D is 1993; 16: 813-820.

2 Jaeken J, M atthijs G, B arone R, Carchon H : Syndrome of the month: carbohydrate-deficient glycoprotein (CDG) syndrome type I. J M ed G enet 1997; 34: 73-76.

3 Jaeken J et al: Sialic acid-deficient serum and cerebrospinal fluid transferrin in a newly recognised genetic syndrome. Clin Chim A cta 1984; 144: 245-247.

4 Stibler $\mathrm{H}$, Jaeken J: Carbohydrate deficient serum transferrin in a new systemic hereditary syndrome. A rch $D$ is Child 1990; 65: 107-111.

5 Wada $Y$ et al: Structure of serum transferrin in carbohydrate-deficient glycoprotein syndrome. Biochem B iophys Res Commun 1992; 189: 832-836.

6 Yamashita K et al: Sugar chains of serum transferrin from patients with carbohydrate deficient glycoprotein syndrome. J Biol Chem 1993; 268: 5783-5789.

7 Clayton $\mathrm{P}$ et al: Carbohydrate deficient glycoprotein syndrome: normal glycosylation in the fetus. $L$ ancet 1993; 341: 956.

8 Stibler H, Skovby F: Failure to diagnose carbohydratedeficient glycoprotein-deficient syndrome prenatally. Pediatr Neurol 1994; 11: 71. 
9 Martinsson T et al: Linkage of a locus for carbohydratedeficient glycoprotein syndrome type I (CDG 1) to chromosome $16 \mathrm{p}$, and linkage disequilibrium to microsatellite marker D 16S406. H um M ol G enet 1994; 3: 2037-2042.

$10 \mathrm{M}$ atthijs $\mathrm{G}$ et al: $\mathrm{E}$ vidence for genetic heterogeneity in the carbohydrate-deficient glycoprotein syndrome type I (CD G 1). G enomics 1996; 35: 597-599.

11 Van Schaftingen $\mathrm{E}$, Jaeken J: Phosphomannomutase deficiency is a cause of carbohydrate-deficient glycoprotein syndrome type I. FE BS L ett 1995; 377: 318-320.

12 Matthijs $G$ et al: Mutations in a phosphomannomutase gene, PMM 2, on chromosome 16 in carbohydrate-deficient glycoprotein type I syndrome (Jaeken syndrome). N ature G enet 1997; 16: 88-92.

13 Jaeken J et al: Phosphomannomutase deficiency is the main cause of carbohydrate-deficient glycoprotein syndrome with type I isoelectrofocusing pattern of serum sialotransferrins. J I nher M etab D is 1997; 20: 447-449.
14 Matthijs $G$ et al: Lack of homozygotes for the most frequent disease allele in carbohydrate-deficient glycoprotein syndrome type IA. A m J H um G enet (in press).

$15 \mathrm{Dib} C$ et al: A comprehensive genetic map of the human genome based on 5264 microsatellites. Nature 1996; 380 Supplement.

16 Schollen $\mathrm{E}$ et al: Comparative analysis of the phosphomannomutase genes PMM 1, PMM 2 and PMM 27; the sequence variation in the processed pseudogene is a reflection of the mutations found in the functional gene. $\mathrm{H}$ um $\mathrm{M}$ ol $\mathrm{G}$ enet (in press).

17 Bjursell $C$ et al: Fine mapping of the gene for carbohydrate-deficient glycoprotein syndrome, type I (CDG 1): linkage disequilibrium and founder effect in Scandinavian families. G enomics 1997; 39: 247-253. 